\title{
Téoros
}

Revue de recherche en tourisme

\section{Portrait des principaux centres de villégiature des Laurentides}

\section{Henri-Paul Garceau}

Volume 15, numéro 1, printemps 1996

Les Laurentides, quelles Laurentides?

URI : https://id.erudit.org/iderudit/1075048ar

DOI : https://doi.org/10.7202/1075048ar

Aller au sommaire du numéro

Éditeur(s)

Université du Québec à Montréal

ISSN

0712-8657 (imprimé)

1923-2705 (numérique)

Découvrir la revue

Citer cet article

Garceau, H.-P. (1996). Portrait des principaux centres de villégiature des Laurentides. Téoros, 15(1), 18-22. https://doi.org/10.7202/1075048ar d'utilisation que vous pouvez consulter en ligne.

https://apropos.erudit.org/fr/usagers/politique-dutilisation/ 


\section{POR'TRAIT des PRIncIPaUX \\ CENTRES DE VILLÉGIATURE DES LAURENTIDES Henri-Paul Garceau}

Henri-Paul Garceau
a oeuvré en hôtellerie pendant
plus de trente-cinq ans à titre
de directeur des
communications de
I'Association des hóteliers de
la province de Québec et de
rédacteur en chef de la revue
Hötellerie et Restauration.
En décembre dernier,
Henri-Paul Garceau a publié
chez XYZ Éditeur,
dans la collection
Toutes latitudes dirigée par
Normand Cazelais, Chronique
de l'hospitalité hôtelière du
Québec de 1940 à 19801.
II y raconte dans le détail
comment, plongés dans la
ugrande noireur du
duplessisme, des individus, des
groupes, des associations ant
permis au Québec de s'afficher
comme un des leaders de la
restauration et de l'Hôtellerie
en Amérique»,
Les extraits qui suivent
dressent un portrait des
principaux centres
cours de cette période

\section{Les principaux \\ centres de villégiatures}

L'hôtellerle de villéglature du Québec s'étalt Installée blen avant la Deuxlème Guerre mondiale, en particuller dans les Laurentides au nord de Montréal, dans I'Estrle, dans quelques zones autour de la ville de Québec, dans certaines villes du Bas-SaintLaurent, dans Charlevolx et en Gaspésle.

C'étalt l'époque où les vacanclers avalent l'habltude de cholsir une destination durant l'été pour y séjourner plusleurs semaines, bien souvent en famille. On y revenalt chaque année, en réservant plusieurs mois à l'avance et parfols immédiatement à la fin du séjour. On s'y rendalt en train ou en automobile, et, dans le Bas-Saint-Laurent et dans Charlevolx, Il y avalt les célèbres crolslères. (...)

Une entreprise hötellère de villéglature se devait de concentrer son chiffre d'affaires annuel durant six à huit mols d'exploltation et cela à des coûts élevés d'entretlen. Vollà sans doute pourquol nous verrons très peu de nouveaux Investilssements dans ce secteur au cours des décennles de l'après-guerre.

La région la plus privlléglée fut sans doute celle des Laurentudes, au nord de Montréal. C'est elle quil eut le moins à souffrlr des fluctuations salsonnières.

Pour les Montréalals et plusieurs milliers de cltoyens de la province de Québec, la réglon des Laurentides étalt un lieu Idyllique: aimalt-on la pêche ou la chasse? Les Immensités montagneuses trouées de milliers de lacs comblaient les appétits des conquérants de la faune. Aimait-on le climat rigoureux de l'hlver, les grands espaces blancs et les côtes poudreuses pour le ski? Plus de cent remonte-pentes automatiques étalent disponibles. Pour d'autres, les Laurentides sont cette source vlve de la beauté naturelle propice à la poésie, à la réflexion. Le grand silence de la montagne. son air pur et ses soleils brûlants inspireront le poète, le peintre ou le musicien.

Pour plusleurs, frlands de bonne chère et de bons vins, les auberges et les hôtels de plaisance regorgealent de spéclalités culinalres et de caves prestigleuses. De falt, on y comptalt plus de 35 établissements qui se signalalent par des spéclalités qui se comparalent avantageusement avec celles des meilleurs restaurants de Montréal.

Avant la guerre, un voyage dans les Laurentides falsalt l'objet de longs préparatifs. Depuls 1960 , avec le parachèvement de l'autoroute qul permet d'atteindre le cour des montagnes en molns d'une heure, plusleurs cltadins y vivent maintenant à l'année longue tandls que nombre d'hommes d'affalres s'y rendent tous les week-ends pour y falre le plein d'oxygène.

\section{Nature et équipements récréatifs}

Lune des belles attractions des Laurentides est sans conteste le parc du Mont-Tremblant. Sa superficle de plus de 3000 kilomètres carrés offre tous les avantages récréatlifs aux amateurs de plein alr: camping, plque-nique, balgnade, canotage, excurslons en forêt que l'on découvre le long de la rivière du Dlable et des lacs Chat et Monroe. Plusleurs rlvlères et plus de 1000 lacs favorisent la pêche.

Ce centre de la nature ne dolt pas masquer le falt qu'au cours des années cinquante la réglon des Laurentides. depuls Saint-Hyppolite près de Salnt-Jérôme jusqu'à MontTremblant, a connu des développements récréatifs et culturels extrêmement coûteux, la plupart financés par des entreprises hôtelières ou du molns encouragés par elles. En 1961, la Laurentian Resorts Assoclation, qui regroupalt les principaux hôtels de villéglature, publlalt les résultats de l'inventaire récréatuf de 15 hôtels dont le total s'élevalt à 5,3 millions de dollars. Cet inventaire comprenait l'aménagement

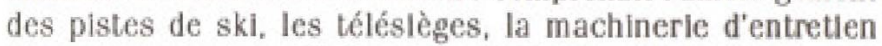
des pentes et de fabrication de la nelge, des curlings, des patinolres, des carrioles, des toboggans; et, pour la salson estivale, des terrains de golf, des courts de tennis éclalrés, des piscines chauffées, des écurles d'équitation, le canotage et le skı nautique, les bateaux à volle, les jeux de croquet, de marelle, de tír à l'arc, les pédalos, le ballon-volant, le golf minlature et quantité d'amusements intérleurs, têls que le billand et le ping pong.

Quand on faisait le décompte de l'investissement global de ces 15 hôtels, c'est-ầ-dire l'ensemble Immobiller y comprls les sources de la restauration, la somme s'élevalt à $18 \mathrm{mIl}-$ llons de dollars. Plusieurs d'entre eux, au cours des années soixante et solxante-dix, effectueront d"importantes amélio- 
rations et des agrandissements, augmentant le nombre de chambres et de salles de congrès, la plupart ayant été constrults avant la guerre. Le seul nouveau centre de villéglature à être développé par la sulte sera l'hôtel Estérel de Fridolin Simard, à Ville Estérel.

SI tous ces hôtels ont réussi à conquérir une clientèle de cholx tant en hiver qu'en été. ils le dolvent à leur préoccupation d'assurer une culsine de haute qualite et un hébergement de grand confort.

\section{Le Mont-Gabriel}

Le premler hôtel des Basses-Laurentides à se développer sera le Mont-Gabriel, perché à 390 mètres d'altatude sur le haut d'une montagne qui surplombe les villages de SaintSauveur, de Pledmont et de Mont-Rolland.

Ce domaine étalt la propriété de Maries-Joséphine Hartford, héritière de la chaîne d'alimentation Atlantic \& Pacinc Stores. En 1935, elle lit construire une auberge en bols rond de 16 chambres, avec une salle à manger et unc salle de sefour.

Après la guerre, le domalne passa aux mains de l'entrepreneur Herb o'Conneli, qui acheta plusleurs terres environnantes pour porter la superflcle à 1400 acres. II fit construlre une plsclne extérleure chauffée de grandeur olymplque et aménagea 15 plstes de $\mathrm{skl}$ desservies par hult remontepentes automatiques alnsi qüun chalet pour les skieurs. Lauberge, qul prit le nom de Mont-Gabrlel Club. fut dirigée par Stanley Ferguson, un des princlpaux promoteurs de skl dans les Laurentides. Cest à cet hôtel que furent jetées les bases du Comité des hôtels de villéglature pour mener les campagnes de promotlon * More and better snow in Québec-

En 1960, lhôtel devint la proprićté de Donald Memford, viceprésident de Hilton Canada et directeur général de l'hôtel Reine Ellzabeth de Montréal. II construlsit l'alle Tyrollenne. un agrandissement de 40 chambres et salons, la section La Marquise comprenant 71 chambres. une salle à manger de 300 places et le Grand Salon pour réunlons et conférences. L'établissement de 145 chambres se donnait une seconde vocation en cultivant la clientèle de congrès.

Les premlers canons à nelge des Laurentides firent leur apparition: Mont-Gabriel devenait le premier centre de ski ha offrir de la neige artificlelle et à eclairer ses pistes de ski lo solr.

$(\ldots)$

\section{Le Chantecler}

A Salnte-Adele, Le Chantecler fut un autre centre important de villeglature. A. B. Thompson avait acquis un domaine de 400 acres en 1915 sur lequel existait une auberge de 35 chambres.

Au cours des années quarante, II effectua plusleurs agrandissements: ajout de chambres, de chalets loués à la salson. salles de réception et de congrès. Létablissement logealt 250 personnes et pouvait servir 1000 personnes à la fois. Les familles bourgeolses de Montréal ainsl que de nombreux
États-Uniens y passalent des vacances de ski ou estivales. profitant des diverses activités sportives et culturelles.

\section{$(\ldots)$}

L'hôtel en plerre des champs enjollvé par des tours conlques évoqualt le style d'un château. Tout autour s'étendatent des parterres de fleurs. $\AA$ quelques pas de l'hôtel, le lac, bordé d"une plage de sable naturel, permettalt la natation. Ia volle et le canotage.

\section{(..)}

Les courts de tennis et le centre d'équitation complétalent l'équipement sportif estival. En hiver, la cllentèle de l'hôtel pouvait benéflcier de trols pentes de ski équlpées de remonte-pentes.

Durant les années cinquante, le Chantecler était l'hôtel de villóglature le plus modeme des Laurentudes. A quelques pas de l'hôtel, un pavillon offralt plusicurs suites de luxe appréclées par les visiteurs de marque el les congressistes. Le Chantecler se signalalt par une culsine françalse de grande réputation. En 1959, la Gourmet Soclety of New York Iul décernait son certificat d'honneur international pour soullgner la qualité de sa table. Ce titre servira de marque de commerce pour l'hơtel. Il sera mis en évidence par George Powell qul depuls 1955 dirigealt l'hôtel à titre de président et directeur général.

Journaliste de carrière et Initié au marketung hôtelier avec la chaîne Sheraton, George Powell fit connaître Le Chantecler par une habile stratégle de communication à travers le Canada et les Etats-Unis. en s'impliquant dans de prestigieuses organisations hôtellères et touristlques. Il fut durant 10 ans prósident du comlté des relations publiques de la Hotel Assoclation of Canada et directeur de la Canadlan Tourist Associatlon. En 1959. Il fut le premier Canadien à occuper la présidence de la Hotel Sales Management Association, un regroupement de 1500 membres recrutés parmi les directeurs d'hôtels et les promoteurs touristlsques à travers l'Ancrique du Nord.

\section{Le Montclair}

Situć dans le village de Sainte-Adèle, lancien Salnte-Adèle Lodoe, populaire durant la guerre à cause de son cabaret fréquenté par les mllitalres en congé, devint en 1957 l'hôtel Montelair sous la présidence de Roger Couillard.

Cet artisté en art graphique doué d'un talent de designer rénova l'établissement en lui donnant le cachet de distinction qu'll méritalt et en révisant de fond en comble tout le servlce de la restauration. Après avoir épuré l'endroit d'une clientèle tapageuse et indésirable, il conquit rapidement la faveur des hommes d'affalres et des professionnels en quête d'une bonne table, grăce à la présence d"un chef remarquable. Cornell Dimitri.

\section{Le Alpine Inn}

A l'cxtrémité du village de Sainte-Adèle, en direction du lac Masson. sélevait le pavillon central du Alpine Inn en bols 
rond et entouré d'un rocher escarpé, d'une piscine, d'une ri= vière poétique, d'un court de tennls et d'un golf miniature. tandis que sur une vaste propriété s'étendalent une vingtaine de chalets en bordure d"un terrain de golf.

Fondée en 1924. l'auberge de 35 chambres avalt été incendlée en 1939 pour être reconstrulte l'année sulvante par un financier montréalals du nom de T. G. Potter. Roland Libolron, qui en étalt contrồleur à cette époque, se souvient que les tarlfs d'hébergement, plan américaln, étaient de 8.50 \$ par jour.

Après le décès de M. Potter en 1946, I'hôtel fut acheté par Vernon G. Gardy, délà propriétaire de l'hôtel Mont-Royal de Montréal et de plusieurs autres en Ontarlo. Lorsqu'il vendit ses Intérêts à Sheraton Corporation en 1950 pour la somme de 4,5 millions, Il conserva le Alpine Inn pour en falre un des joyaux de villéglature des Laurentides. Roland Libolron fut nommé vice-président et contrôleur de l'établissement et demeura le chargé d'affaires de Vernon Cardy.

Jusquen 1960 , le complexe subit dimportantes transformatlons. Il devenalt le seul hôtel de villéglature au Canada à être entièrement climatisé. Par son architecture et son ambiance, le Alpine Inn Incarnait le caractère authentique des grands hôtels de montagne. Son hall d'entréc, avec son Immense foyer en plerre des champs, et les murs en bols rond, la salle à manger d'une capacité de 350 personnes dotée de fenêtres panoramiques sur trols côtés suscitatent l'admiration du visiteur et la jole des barons de la finance et du jet-set américain devenus à cette époque des clients réguliers de l'hôtel.

Graduellement, le développement de ce domaine de 2000 acres se poursulvit par la construction d'un pavillon pour un curling, l'installation d'une piscine intérieure et d'une boutlique de sport. Au sous-sol de l'hôtel furent inaugurés une rótisserie, un bar et des salles de congrès. C'est au niveau de la restauration que le Alpine Inn se créa une réputation exceptionnelle.

\section{(...)}

\section{L'Estérel}

Le seul grand développement de villéglature à être entrepris dans les Laurentides au cours de la période de 1950 à 1980 sera celui du domaine Estérel, par les frères Fridolin et Thomas Simand. Estérel est le nom d'un massif enchanteur de Provence, lequel, dit-on, évoque l'idéal d'un paradis terrestre.

Cet endroit avalt déjà séduit un personnage mystérieux, parfols suspect à cause de ses affinltés avec le parti nazi, le baron Empain, qui avalt cu l'idée de construlre un ensemble Immobiller d'envergure. La construction d'un hôtel de luxe, d'un centre commercial, d'un club sportif et d'un théâtre fut. interrompue par la guerre alors que ses blens furent confisqués. Un peu plus tard, l'ensemble fut ravagé par un incendie.

En 1959, les frères Simard décldèrent de ressusciter l'Estérel en incorporant le domaine en munlcipalité. Ils entreprirent des travaux d'aqueduc, d'électrification. l'instal- lation du service téléphonique, et construisirent 35 kllomètres de routes pavées, un parcours de golf de 18 trous, une cabane à sucre, des courts de tennis, tandls que le lac olfralt toutes les possibilités de sport nautique.

Pour compléter ce développement ambitleux, Ils construIsirent l'hôtel Estérel, un superbe monument de 125 chambres, munies de balcons imitant la passerelle d'un navire, qul s'avance dans les eaux du lac Dupuls. Les plans de l'hôtel, préparếs par l'architecte Jean-Guy Clément, comprenalent plusleurs salles de conférences pour 600 personnes, deux vastes salles à manger, un bar-salon, une plscine intérleure, un gymnase et un service d'ascenseurs.

Lorsque I'hôtel ouvrlt en 1964, Il constitualt une nouvelle attraction touristique majeure dans la réglon des Laurentides. A l'instar de ses ainés établis depuls plusleurs années, Il se distinguera par sa cuisine de qualité dirlgée par le chef Rudl Schneider. La salle à manger avalt un cachet de haute distinction grâce à la galerie de pelntres canadiens qul y exposalent leurs ceuvres. Le service. longtemps dirı́gé par Jules Rolseux, un sommeller et maitre d"hôtel original, rehaussalt l'ambiance.

Toujours en quête d'innovation. Fridolin Simard caressalt depuls longtemps le projet d'établir à son hôtel une station cllmatique à l'europécnne. Ses voyages dans les statlons d'Europe et des États-Unis l'avaient persuadé qu'une telle initlative en villéglature viendrait satisfalre les besoins des vacanclers.

En 1974, II Inaugurait ce nouveau service qui constutualt une premlère dans les hôtels de villéglature du Canada. On la disait comparable aux melleures stations climatiques au monde, y compris celle de Mabella en Espagne, fréquentée par des millardalres, Les gens en quête de reconditionnement physique ou de désintoxication n'auralent pas à voyager en Europe ou aux États-Unis. Le coût d'investissement pour assurer les traitements à l'aérosol. la cavitation, l'électro-théraple, les balns d'algues, les douches sousmarines, les bains de mousse, les massages et les soins esthétiques s'élevait à 250000 dollars. Cecl portait à $15 \mathrm{mll}-$ lions les coûts de développement du domalne Estérel.

\section{La Sapinière}

Au rang des hôtels de vllléglature ma|eurs, La Saplnière de Val-David s'afirme par son caractère distinctuf quil ne souffre aucune comparaison. En 1980, Il comptait déjà 45 ans đ'existence assurés par les mêmes propriétaires, Jean-Louls Dufresne cl son épouse.

Jean-Louis Dufresne appartlent à cette race de défricheurs qui ont franchi les frontières de Salnt-Jérồme, vers 1850. pour falre lcur nld dans la réglon de Sainte-Agathe. II tlent d'eux la qualité propre aux plonnlers: la ténacité, celle qui permet de ne Jamals abandonner, de demeurer fidèle au patrimoine durement acquis.

Au cours de son existence, l'établissement a toujours été en perpétuel changement. Par exemple, entre 1960 et 1970. toutes les chambres ont été refaites, plus spacieuses el plus luxueuses, et des suites avec salon pour les réunlons de petits comités furent aménagées. 
En comparant la photo du premler hôtel de 20 chambres avec celle de 1980 , Il est difflclle de retracer ce qul existalt au début. Le hall de réception. la salle à manger, la cave à vin où dorment quelque 25000 boutelles, les chambres de grand luxe, le bar-salon Quatre Solells, les salles de con= férences, la culsine, la buanderie ainsl que l'aménagement. extérleur avec son vaste stationnement, la plscine et de superbes parterres de fleurs sont autant de services ajoutés par étapes pour le confort et la détente de la clientèle.

Ge n'est pas sans raison que l'auberge fut la premlère au Canada à être admise dans la prestigleuse chaine hôtellère Relals et Chăteaux, car sa renommée repose sur l'excellence de sa table et son caractere françals.

\section{$(\ldots)$}

Consıdéré comme l'Idéal de l'hôtellerle famlllale, La Saplnière fut l'endrolt - jusqu'à ce que l'Institut de tourisme et d'hôtellerie du Québec ouvre ses portes - oú se sont tenus les cours de perfectionnement dispensés par le Service d'hôtellerle du Ouebec. G'est là que l'on pouvait côtoyer un personnel qualifié, fler de son appartenance et totalement déwoué à l'entreprise.

\section{$(\ldots)$}

\section{Le Gray Rocks}

Dans les Hautes-Laurentides, deux hôtels rettennent notre altentlon: le Gray Rocks et le Mont-Tremblant Lodge.

Le Gray Rocks est l'ancêtre des hôtels de villéglature des Laurentides. Dabord une humble auberge de 10 chambres, établie en 1906 par Ernest et Lucille Wheeler à l'Intention des chasscurs et des defricheurs, I'hôtel sera agrandi au fil des ans par lours fils Tom et Harry. Après la guerre, Il se posiltonnera comme centre de ski et site de villéglature estival recherche. Son expansion sera rapide sur un terrain de 1700 acres sur les bords du lac Oulmet à Saint-Jovite.

Au début des années quarante, l'hôtel offrira 85 chambres dans un pavillon central entouré de dépendances, solt sept malsons pour loger le personnel et 12 chalets accommodant de quatre à 12 personnes. DLX ans plus tard, II sera agrandl pour offrir 150 chambres, des salles de conférences et un equipement récréologique pour les vacanclers d'hiver et d'ćlé.

Le Gray Rocks devenall une institution autosulfisante avec sa propre plste d'atterrissage, son service réguller d'aviation, sa ferme d'élevage et de culture, son immense caveau à légumes, son terrain de golf, son centre d'équitation. ses courts de tennis, et un lac propice à la natation et au bateau à volle.

Il réussissalt à malntenir un personnel permanent douze mols par annéc. Plusleurs cmployés avalent plus de 20 ans de servlce. Dans un mémoire présenté à la municipalité de Salnt-Jovlte, Il etalt demontré que le Gray Rocks assurait la subsistance à plus de 250 personnes de la localité.

Vers 1950, environ 100000 ELats-Uniens et Canadiens avalent fróquenté le Gray Rocks, soit pour des vacances esti- vales, soit pour des excursions de chasse et de pểche en automne, solt pour le ski en hiver.

Les succes et le rapide développement du Gray Rocks s'expliquent précisếment par sa versatllité qul lul permettait d'attirer une clientèle étalée sur trols salsons.

La compagnle d'avlation Wheeler Alr Line, Inaugurée par Tom Wheeler après la Premlère Guerre mondlale, fut la première entreprise de transport commerclal par avlon au Canada. Ce service sera mis à la disposition des amateurs de pêche et de chasse désireux d'aller dans le Grand Nord et attrera pendant plusleurs décennles une riche cllentèle ctats-unienne.

Le second lacteur de succès réside dans une organlsation du skl blen structurée, grâce à son école de ski Snow Eagle. Dirlgée au début par l'Instructeur autrichien Herman Gadner, puis par Hans Falkner, elle sera prise en charge par Réal Charette à partir de 1948. A l'automne de chaque année, Réal Charette visitalt les clubs de skl de New York, de Washington, de Rochester. de Detrolt et de Toronto et donnaiti des conférences illustrées par un film sur les méthodes de ski cnsclgnécs par son ćcole.

\section{$(\ldots)$}

En 1980, le Gray Rocks possédalt des structures opérationnelles a administratives dynamlques sous la direction générale d'Yves Pinard.

Avec ses 230 chambres, ses nombreuses salles de réunlon. sa vaste salle à manger, sa culsine fonctionnelle dirigé par Jean Labelle, qui avait fait ses classes avec Plerre Demers du Ritz-Carlton, son centre de skl de 18 plstes, de trols Léléslèges et de deux télébars, son service d'avlon, son golf de 18 trous, ses 22 courts de tennis, sa marina pour ski nautique et bateau à volle, son territoire privé de 21 lacs, tout cet equipement hôtelier lui garantissait une clientèle fldèle annce après année.

Ce qul le différenclait sur le marché des vacances, c'est son visage humalin caractérise par l'animation et un accuell personnalisé. En somme, le Gray Rocks ne vendalt pas un hốtel, mals des vacances de rêve.

(..i)

\section{Le Mont-Tremblant Lodge}

A quelques kllometres du Gray Rocks se dresse le majestucux mont Tromblant quil domine, à 900 mètres d'altitude, les lacs, les vallées et les autres montagnes envlronnantes. Blen avant la venue des colonisateurs, les Amérindiens avaient été fascinćs par cette montagne qui Incarnalt pour cux le dieu protecteur de la forét, Manitou Ewitchi-Saga

On se souvient qu'au début de la Deuxtème Guerre mondiale le financler américain Joe Ryan, en vacances au Gray Rocks, avalt escaladé le faîte de cette montagne et décidé d'en faire lo plus prand centre de ski de l'Amérique du Nord.

En moins d'un an la piste de ski s"ouvrit et les tours de pomontés mécaniques furent érigées. Le premter télésiège 
de 1400 mètres fonctionnalt. Le sommet du mont. Tremblant devenalt accessible pour les compétitions internationales. tandis qu'à ses pleds surgissait comme par enchantement une hôtellerie pavillonnaire qui reflétalt par son architecture et son design intérleur le caractère de nos anciennes malsons munles d'un tolt en pente et de lucarnes. Quatrevingt-quinze bâtiments occupalent le flanc de la montagne: le pavillon central. le chalet des skieurs, la malson du personnel, la boutlque de sport, le salon de beauté et la plscine.

Joe Ryan et son épouse Mary étalent de fervents catholiques. Aussi. Ils firent construlre la chapelle Saint-Bernard, répllque d'une égllse du vlllage Salnt-Laurent à l'île d'Orléans, qul datalt de 1670.

En 1965, l'empire du Mont-Tremblant Lodge fut acquis par un syndicat de trols financiers montréalais composé de JeanLouis Lévesque, de Roger Beauchemin et d'André Charron. Ils flrent appel à un gestionnaire d"expérlence dans les hôtels de villéglature en la personne de Stanley Ferguson. Originalre de Salnte-Agathe-des-Monts, celul-cl s'etait Initié à l'hootellerie de montagne au Laurentide Inn et fut par la sulte directeur du Mont-Gabriel. Durant ces séjours dans les Laurentides, Il avalt été un promoteur remarquable des campagnes de ski et présıdent de la Laurentian Resorts Assoclation.

À son arrivé, le Mont-Tremblant Lodge offrait 90 kilomètres de plstes de ski, trols télébars, deux télésièges et une téléchalse double, un équipement évalué à un million de dollars.

Une école de skl, dirlgée par Ernle McCulloch, employali 30 instructeurs diplômés de l'Alliance canadienne des montteurs de ski. Le regretté Joe Ryan, décédé dans un accident en 1950, avalt lalssé un héritage incomparable dans le développement du skı dans les Laurentıdes. Grâce à ce développement qul représentalt un investissement de six milllons de dollars, la station Mont-Tremblant étalt devenue le porte-étendard qul plaçalt la réglon au rang des centres internationaux.

Mais, si le ski demeurait l'atout majeur de cette entreprise, sa réputation auprès du tourisme estival et des hommes d'affaires désireux d'y tenir des congrès suscltalt autant d'intérêt. Stanley Ferguson procéda à une réorganisation des lieux pour en faire également un centre de vllléglature estival et un centre de congrès.

C'est aussi sous sa gouverne que le Mont-Tremblant Lodge décida d'ouvrir toutes grandes ses portes à la clientèle canadienne-françalse. Lancienne direction s'étalt montrée plutôt sectalre de ce polnt de vue. On avalt l'1mpression que le Mont-Tremblant Lodge était réservé à l'aristocratie anglosaxonne et sl par hasard un visiteur anonyme s'y présentait Il était recu froidement par le personnel.

Au cours des années solxante-dix, tout en conservant sa clientèle attirée par les sports d'hiver - dont $70 \%$ provenait des États-Unis et $30 \%$ de l'Ontario et de la réglon de l'Outaouais - le Mont-Tremblant Lodge attiralt en cté une Importante clientèle québécolse.
Avec sa plsclne climatisée, son golf, ses quatre courts de tennls, sa plage sur le lac, de nombreuses activltés sportives et soclales furent organisées: cliniques de natation, de tennis, d'équitation, de golf, excursions et plque-nlques en montagne. montées en téléphérique au sommet du Mont Tremblant.

En quelques annees, la clientèle estivale avalt doublé et l'hôtel employalt un personnel de 190 personnes, tandis qu'en hiver 250 y travalllalent. La masse salarlale annuelle étalt de deux millions de dollars.

Lorsque Stanley Ferguson quitta le Mont-Tremblant Lodge en 1977. Il lalssait un domaine de 116 bâtıments logeant 211 chambres et une salle à manger de 200 places.

\section{(...)}

\section{Les grands hôtels: une valeur sure}

Tous ces grands hôtels de villéglature que nous venons de clter constituent en sol des attractions majeures et permanentes qui favorisent la venue des touristes au Québec. Très peu d'entre eux disparaîtront et Ils continueront à s'améliorer au rythme du changement des gouts de la cllentèle et des exigences des nouvelles technologles.

Autour d'eux se grefferont des auberges, des restaurants, des terrains de camping, des bases de plein air qui compléteront (ces) infrastructures dhospitallté.

Notes

1 GARGEAU, Henri-Paul, Ghronique de Thospitaine bókelière du Ouchec de 1940 à 1990, XYZ Éditeur, collection Toutes latitudes, Montréal 1995, 239 papes. 